\title{
The Simulation and Analysis of Erosion to a Layered Structure of a Single Particle
}

\author{
Ping LIANG ${ }^{1, a}$, Yi-Bo XU1 ${ }^{1}$, Wen-Mo LI ${ }^{2}$ and Lu-Quan REN ${ }^{1}$ \\ ${ }^{1}$ Key Laboratory of Bionic Engineering (Jilin University), Ministry of Education, Changchun, China \\ ${ }^{2}$ College of Materials Science and Engineering Jlin University, Changchun, China \\ aliangping@jlu.edu.cn
}

Keywords: Dorsal scale, Layered structure, Stress wave.

\begin{abstract}
The dorsal scale of Laudakinstoliczkanain desert has good performance of erosionresistance because it is composed of protein "soft" layer and the covering of the cuticle "hard" layer. In this paper, we simplify it to a layered structure called a bionic layered structure(BLS). Based on it, a numerical model for a single particle to impact BLS is established for simulating the impact process. Analysis results shows that BLS can effectively weaken the incident stress wave amplitude, to prevent the failure or damage of the whole structure.
\end{abstract}

\section{Introduction}

Erosion wear is widely existed in machinery, energy, chemical industry, aerospace, and many other fields. The damages can be seen everywhere, e.g. the service life of propeller blades of the aircraft can be dropped to one-tenth the original when flying in dust weather due to a serious erosion of grains of sand[1]. A lot of enormous losses of erosion wear are brought to us. Therefore it is very important to avoid or reduce the erosion damage.

With the development of bionic science, inspired by the biological in nature, a new biological way of erosion-resistance is studied. Gao et al. [2] found that desert lizard body surface possesses very strong erosion-resistance ability due to the surface morphology, structure of interface layer and material gradient. And, these factors can change the stress wave propagation modes (such as different interface reflection and transmission), and stress wave reflection unloading in a certain extent can change the stress distribution of the surface structure. Han et al.[3] also obtains a similar conclusion aiming at desert scorpion.

Desert lizard scales has a hierarchical structure, from the outside to the inside corner: cortex, $\beta$ cuticle, middle, $\alpha$ - cuticle and germinal layer (Figure 1). The cuticle outside is the relatively hard, can well resist the physical impact, on the other hand, the connective tissue is soft, filling in the horny shell below the cuticle. This kind of structure can helps to reduce the erosion of the desert sand.

In this paper, we simplify it to a layered structure called a bionic layered structure(BLS). Based on it, a numerical model for a single particle to impact bionic layered structure is established to simulate the impact process. The stress wave propagation law in BLS is studied using stress wave theory to analyze the damage or failure of material and erosion-resistance mechanism of Laudakinstoliczkana. 


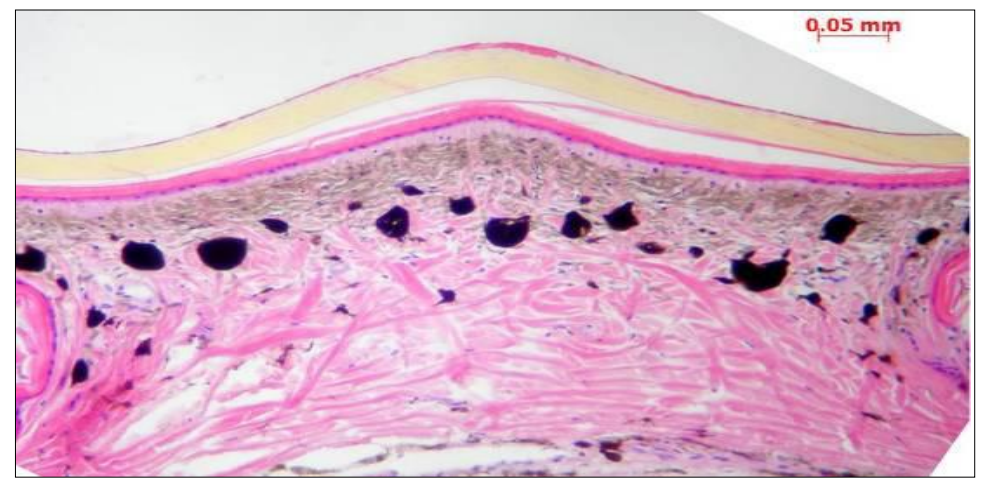

Fig. 1.Structure of desert lizard scales

\section{Introduction of Stress Wave Theory [4]}

Only consider one-dimensional stress wave theory. The equation of one dimensional stress wave is:

$$
\left(\partial^{2} u / \partial t^{2}\right)=c^{2}\left(\partial^{2} u / \partial x^{2}\right) c^{2}=E / \rho
$$

Here $u$ is the particle displacement, $\rho$ the density of material, $E$ elastic modulus and $c$ wave velocity.

From equation (1), the stress wave propagation is decided by $\mathrm{c}$ or wave impedance $\rho c=\sqrt{E \rho}$. In this paper, we say some material "hard" if the wave impedance is bigger or "soft" if the wave impedance is smaller. Stress wave propagates in the medium; the medium interface can produce a series of reflection and refraction. When elastic wave propagates from one medium into another different medium, reflection disturbances and (or) transmission disturbances will be produced.

Use $\sigma_{I}$ to denote the incident stress, and use $\sigma_{R}, \sigma_{T}$ denote the reflection stress and the transmission stress respectively, then we have the following equation:

$$
\left\{\begin{array}{l}
\sigma_{T}=\frac{2 A_{2}(\rho C)_{2}}{A_{1}(\rho C)_{1}+A_{2}(\rho C)_{2}} \sigma_{I} \\
\sigma_{R}=\frac{A_{2}(\rho C)_{2}-A_{1}(\rho C)_{1}}{A_{1}(\rho C)_{1}+A_{2}(\rho C)_{2}} \sigma_{I}
\end{array}\right.
$$

From equation (2), we have

1) If $A_{1}(\rho C)_{1}<A_{2}(\rho C)_{2}$ then $\sigma_{R}$ and $\sigma_{I}$ have the same direction, the incident stress wave propagates from soft materials into hard materials. And if $A_{2}(\rho C)_{2}=\infty$, then $\sigma_{T}=2 \sigma_{I}, \sigma_{R}=\sigma_{I}$, the elastic wave can be viewed as reflected in the free end.

2) if $A_{1}(\rho C)_{1}>A_{2}(\rho C)_{2}$ then $\sigma_{R}$ and $\sigma_{I}$ have the opposite direction, the incident stress wave moves from hard materials into soft materials, soft materials can slow down the stress wave propagation. And if $A_{2}(\rho C)_{2}=0$, then, $\sigma_{T}=0, \sigma_{R}=\sigma_{I}$, the elastic wave can be viewed as reflected in the free end.

\section{The Numerical Simulation}

\section{Finite Element Model of BLS}

Only consider the longitudinal wave, neglect the effect of particle shape. Collision particle is a ball of radius $2.5 \mathrm{~mm}$. As a target, BLS is a cylinder of radius $10 \mathrm{~mm}$. The cylinder consists of three layers stacked together (Figure 2): each layer thickness is $5 \mathrm{~mm}$. The first and the second are "hard" layer (taken as typical carbon steel) and the bottom layer is a "soft" layer taken as rolling pure copper, rolling pure aluminum, or typical rubber etc. in order to investigate the stress wave propagation behavior according to the material of different impedance. Material properties show in 
table 1.

Particle's incident angle of $90^{\circ}$ at center of up surface, the incident speed of $5 \mathrm{~m} / \mathrm{s}$. Total analysis step period is $10 \mu \mathrm{s}$.

Table 1. Parameters of the experimental program

\begin{tabular}{|c|c|c|c|c|}
\hline Element & Poisson ratio & Modulus E (10 $\mathbf{~ M P a )}$ & Density $\boldsymbol{\rho}\left(\mathbf{1 0}^{-\mathbf{9}} \mathbf{g} \mathbf{m}^{\mathbf{3}}\right)$ & $\mathbf{I m p e d a n c e} \sqrt{E \rho}$ \\
\hline $\begin{array}{c}\text { Typical } \\
\text { carbon steel }\end{array}$ & 0.28 & 2.0 & 7.8 & 3.95 \\
\hline $\begin{array}{c}\text { Rolling pure } \\
\text { copper }\end{array}$ & 0.31 & 1.08 & 8.9 & 3.1 \\
\hline $\begin{array}{c}\text { Rolling pure } \\
\text { aluminum }\end{array}$ & 0.32 & 0.68 & 2.7 & 1.35 \\
\hline $\begin{array}{c}\text { Typical } \\
\text { rubber }\end{array}$ & 0.47 & 0.000078 & 0.93 & 0.0085 \\
\hline
\end{tabular}

\section{The Numerical Simulation Results}

Use software of ABAQUS, meshing BLS, setting boundary condition(Figure 2), gets some results. The profile of numerical simulation results(stress nephogram) are shown in Figure 3.

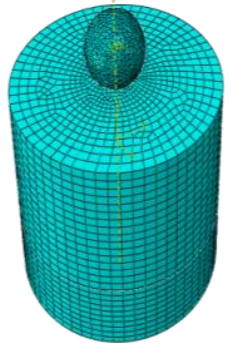

Fig. 2. Grid of BLS

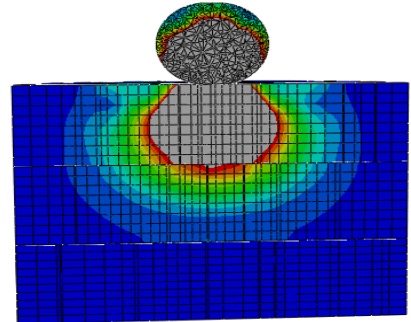

Fig. 3. Stress nephogram

In order to explain the stress wave propagation laws, select 6 observation points from top to bottom along the shaft centerline. Point $\mathrm{A}$ is in collision point on the surface. Points $\mathrm{B}$ and $\mathrm{C}$ are near the combination of top two layers: $\mathrm{B}$ in top layer and $\mathrm{C}$ in middle layer. Point $\mathrm{D}$ is the center of middle layer. Points $\mathrm{E}$ and $\mathrm{F}$ are near the combination of bottom two layers: $\mathrm{E}$ in the middle layer and $\mathrm{F}$ in soft material.

The underlying soft materials according to the typical carbon steel, pure copper, rolling pure aluminum and typical rubber, we use color black, blue, red, green to sign it respectively, i.e. the color of each stress curve. The stress-time curves of 6 selected points are shown as follows (Figure 4A-F).

Let's give an analysis for the results as follows.

For point A, at the initial collision moment, the normal stress wave is just starting; wave forms of four groups of experiments are almost the same. Easily can be seen that particle collision produce a principal stress curve of about $6 \mu$ s duration similar to sine curve. The peak value is about $400 \mathrm{~N}$ and characterized by compressive stress. After the main pressure pulse peak is a waveform similar to sine curve with a disturbance of residual amplitude less than $25 \mathrm{~N}$. Although the peak of principal stress reached $400 \mathrm{~N}$, but the duration is very short and the scope is limited to small areas near collision point. Along to the internal depth of target direction, the stress also increased quickly with the depth. So can speculate that the collision point target material may peels in a small scale due to the high impact load and the tensile stress in adjacent small areas will intensify the process, but for the BLS, damage is only limited to a small local skin layer, will not cause a wide range of material 
failure(Figure 4A).

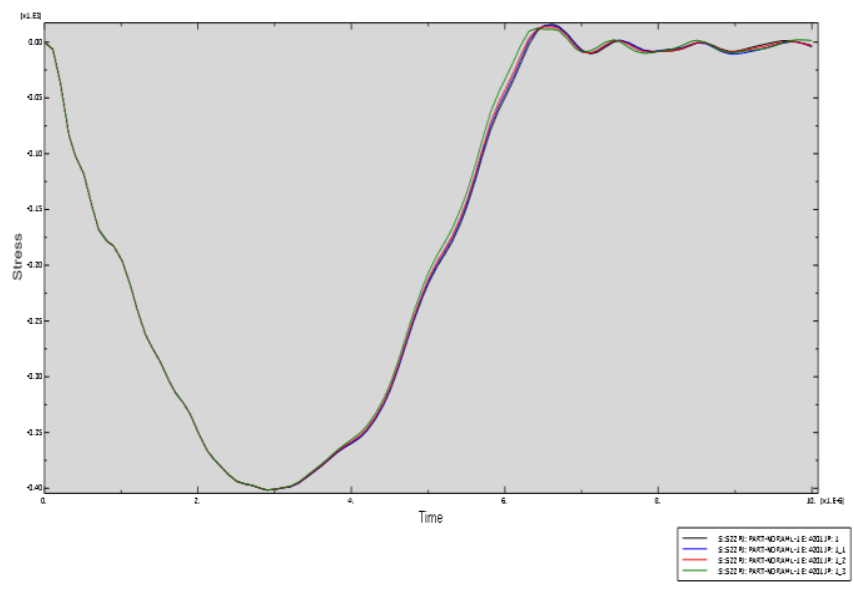

Fig. 4A. The stress wave plot of point A

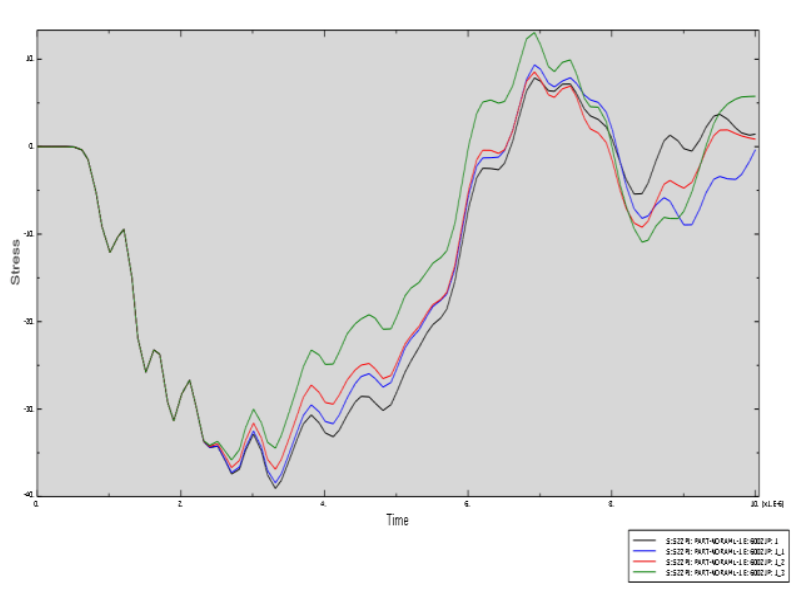

Fig. 4B. The stress wave plot of point B

For points $\mathrm{B}$ and $\mathrm{C}$ in figure 3, the principal stress waveforms separate with different "soft" material. After the peak, the compressive stress of the vibration of residual vibration amplitude less than $15 \mathrm{~N}$ decreases with the drop of impedance, and the speed of attenuation increases (waveform tangent slope increases). From stress wave theory, the stress wave from the materials in "hard" incident "soft", will produce the reflection of the unloading disturbance. Unloading disturbance that before the incident stress amplitude value is reduced, and wave impedance ratio of two layers of material, the greater the reflection the stronger the unloading disturbance, more amplitude reduced. In addition, the principal stress of $\mathrm{B}$ and $\mathrm{C}$ before the peak has a zero value interval(about $0.85 \mu \mathrm{s})$. The reason is: $B$ and $C$ have been not disturbed yet in that time interval(Figure $4 B$ and $4 C$ ).

Compared with points B and C, point D is closer to the interlayer interface of "soft" and "hard" layers. Waveforms separate more apparently(Figure 4D).

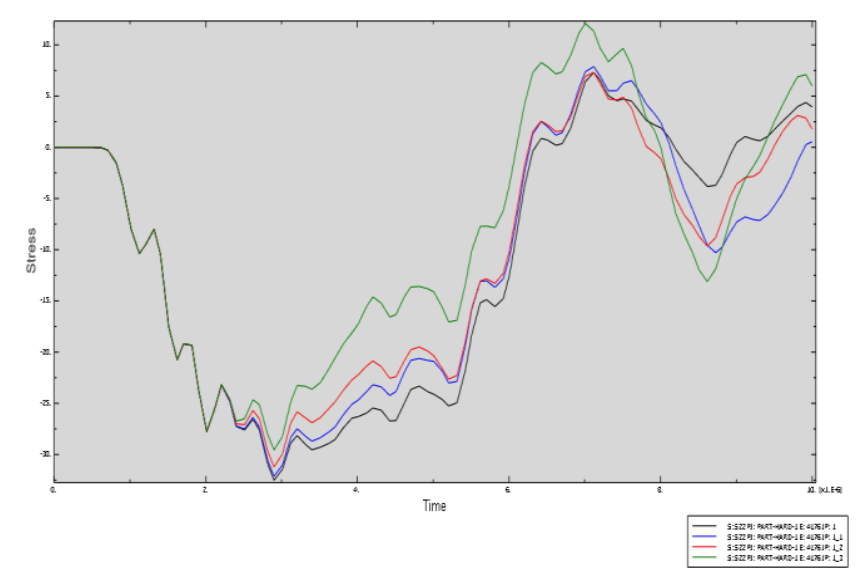

Fig. 4C. The stress wave plot of point $\mathrm{C}$

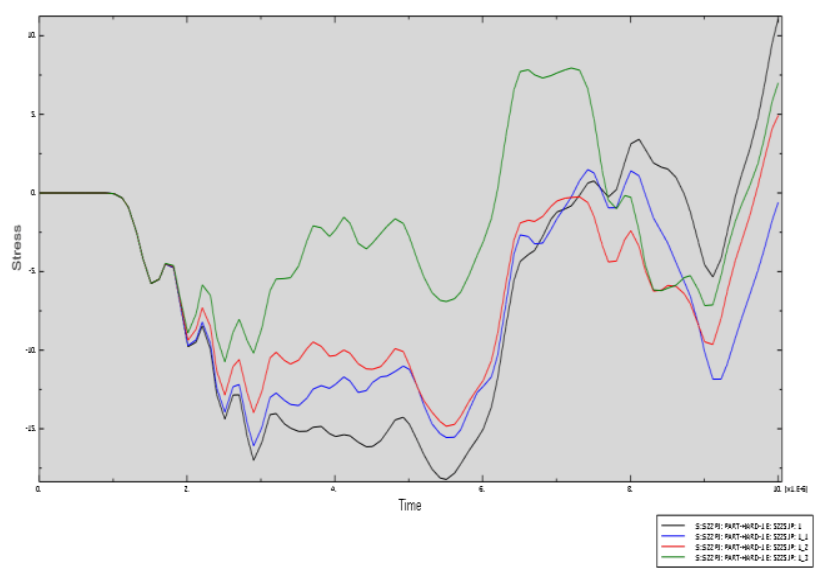

Fig. 4D. The stress wave plot of point D

Take points B, C and D together, we can see that principal stress value in "hard" material also decreases with the reduction of the underlying material wave impedance.

For point E, measurement point of "hard" layer material in the interface. It can be seen that the peak of principal stress has an obvious delay; this is because unloading disturbance has start work on the front of stress wave. And as the underlying material rubber reflects a strong unloading wave, almost all the pressure wave is unloaded to zero(Figure 4E). 


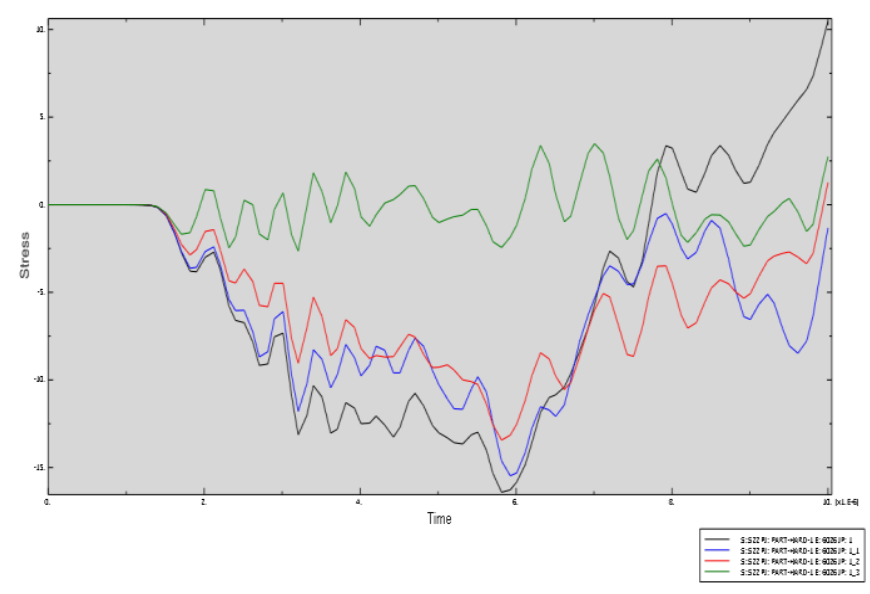

Fig. 4E. The stress wave plot of point E

For point F, in the "soft" layer, we can found the stress level inside of the rubber is almost zero. Stress wave propagation velocity in rubber is very low; particle vibration amplitude is also very small, rubber medium almost "blocking" the spread of stress wave in the internal. At the same time large deformations can occur under the action of stress. Convert the particle kinetic energy is converted into the strain energy in order to achieve sustained release stress effect(Figure 4F).

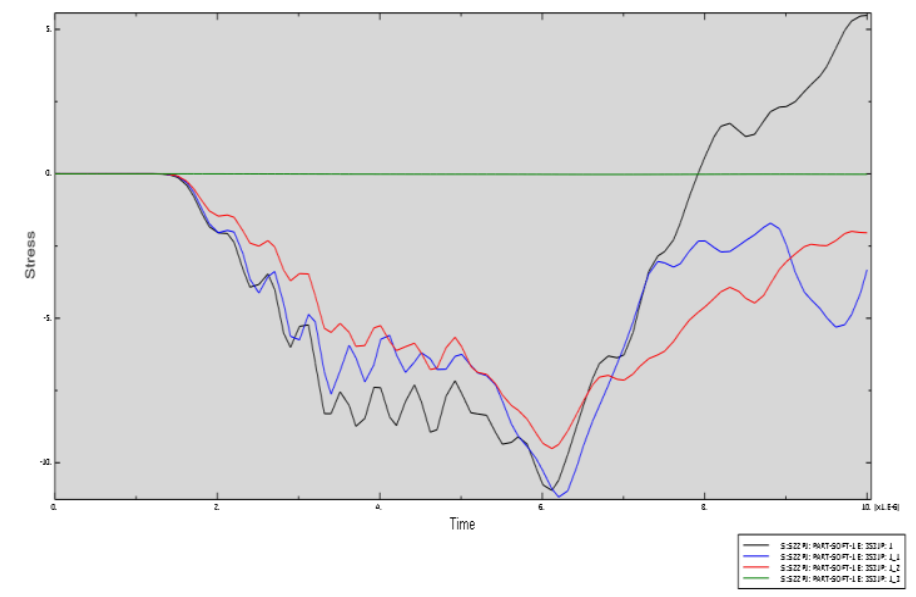

Fig. 4F. The stress wave plot of point $\mathrm{F}$

\section{Conclusions}

In this paper, we take a Laudakin stoliczkana body surface structure as biological prototype, and on this basis simplify it to a bionic layered structure. Further, erosion-resistance of a Laudakin stoliczkana is simplified to a collision problem of a particle to a BLS. Use stress wave theory and ABAQUS soft a numerical simulation and analysis is set up. Some conclusions are obtained as follows:

1) When a particles collides with a BLS on the surface, stress of high peak will be produce, but a very short duration and the influence scale is also small. So, the injury or peeling near the collision area of small scale is possible, and the tensile stress of the adjacent area surface may intensify this process, but won't cause a wide range of material failure.

2) The wave impedance ratio of "soft" and "hard" layer materials has a key effect on the stress wave propagation in the BLS. The wave impedance of soft material is smaller, the stronger unloaded stress wave in interface, more be unloaded the incidence principle stress wave.

3) Microelements inside of the material are in dynamic balance due to the stress wave propagation hard to observe in the naked eye. Stress wave in collision lead to tensile stress, the stress itself also is repeated the process of loading-unloading. Assume an incident particle impacts 
on the surface more severely. If high stress levels continues for a relatively long time and the stress amplitude value is very high, then the plastic deformation is easily generated, Considering Bauschinger effect, tensile stress wave followed by compression stress wave is likely make a whole material become invalid.

From 1)-3), we can see that soft and hard layered structure of desert lizard can helps it reduce the erosion of the desert sand.

\section{Acknowledgments}

This research was financially supported by Science and Technology Development Project of Jilin Province (20150519007JH and 20150101020JC).

\section{References}

1. Li S. Z., Dong X. L. Erosion wear and fretting wear of materials (Beijing, 1993)(in Chinese).

2. Gao F., Ren L. Q. \&Huang H., Biology coupling characteristics of anti-erosive wear of desert lizard's skin. J. Transaction of the Chinese Society for Agricultural Machinery, 40, 180-183(2009) (in Chinese).

3. Han Z. W., Zhang J. Q., Ge C., Erosion resistance of bionic functional surfaces inspired from desert scorpions, J. Langmuir, 28, 2914-2921(2012).

4. Stress wave basic introductory tutorial, Northwestern Polytechnical University press 2007(Xi'an, in Chinese). 